\title{
Statistical Inferences for Lomax Distribution Based on Record Values (Bayesian and Classical)
}

\author{
Parviz Nasiri \\ University of Payame Noor, Tehran, Iran \\ Saman Hosseini \\ University of Payame Noor, Tehran, Iran
}

Follow this and additional works at: http://digitalcommons.wayne.edu/jmasm

Part of the Applied Statistics Commons, Social and Behavioral Sciences Commons, and the Statistical Theory Commons

\section{Recommended Citation}

Nasiri, Parviz and Hosseini, Saman (2012) "Statistical Inferences for Lomax Distribution Based on Record Values (Bayesian and Classical)," Journal of Modern Applied Statistical Methods: Vol. 11 : Iss. 1 , Article 15.

DOI: $10.22237 /$ jmasm/1335845640

Available at: http://digitalcommons.wayne.edu/jmasm/vol11/iss1/15

This Regular Article is brought to you for free and open access by the Open Access Journals at DigitalCommons@WayneState. It has been accepted for inclusion in Journal of Modern Applied Statistical Methods by an authorized editor of DigitalCommons@WayneState. 


\section{Statistical Inferences for Lomax Distribution Based on Record Values (Bayesian and Classical)}

\section{Cover Page Footnote}

The authors are thankful to the referees and editor for their valuable comments. This work was partially supported by the Tehran Payame Noor University (PNU) through a grant. 


\title{
Statistical Inferences for Lomax Distribution Based on Record Values (Bayesian and Classical)
}

\author{
Parviz Nasiri Saman Hosseini \\ University of Payame Noor, \\ Tehran, Iran
}

A maximum likelihood estimation (MLE) based on records is obtained and a proper prior distribution to attain a Bayes estimation (both informative and non-informative) based on records for quadratic loss and squared error loss functions is also calculated. The study considers the shortest confidence interval and Highest Posterior Distribution confidence interval based on records, and using Mean Square Error MSE criteria for point estimation and length criteria for interval estimation, their appropriateness to each other is examined.

Key words: Lomax distribution; record values, maximum likelihood estimation, method of moment, Bayesian estimation, shortest interval, highest posterior density (HPD) interval, quadratic loss function, squared error loss function, prior density, posterior density, simulation, MSE.

\section{Introduction}

Let $X_{1}, X_{2}, X_{3}$, be a sequence of independent and identically (iid) random variable with cumulative distribution (cdf) function $F(x)$ and probability density (pdf) $f(x)$ For $n \geq 1$ define

$$
\begin{aligned}
T(1) & =1, T(n+1) \\
& =\min \left\{j: X_{j} \geq X_{T_{(n)}}\right\} .
\end{aligned}
$$

The sequence $\left\{X_{T(n)}\right\}_{n=1}^{\infty}$ is known as an upper record value statistic and the sequence $\{T(n)\}_{n=1}^{\infty}$ is known as a record time sequence (Arnold, Balakrishnan \& Nagaraja, 1998). Chandler (1952) was one of the first to study record theory and he defined a mathematical model for record values. Record values arise naturally in many applications involving data

Parviz Nasiri is an Associate Professor in the Department of Statistics University of Payame Noor, 19395-4697 Tehran, Iran. Email him at: Pnasiri45@yahoo.com. Saman Hosseini is a Lecturer in Department of Statistics. Email him at: S.hosseini.stat@gmail.com. relating to weather, sports, economics and life testing studies. Many authors have studied records and their associated statistics as well as inference-based testing on records. Some of the best examples may be found in the works of Balakrishnan, Arnold, Nagaraja (1998), Ahsanullah (1998) and Nevzoroz (1946).

Sevgi, et al. (2005) examined the relationship between order statistics and records. Mohammad (2002) and Balakrishnan (1994) examined the recurrent relations between the moments for the generalized exponential and Lomax distributions. Ahsanullah (1974) studied record values received from Lomax distribution, and Ahsanullah and Holland (1994) discussed both scale and location estimation of the distribution of generalized extreme values based on records. Asgharzadeh (2009) discussed both MLE and Bayesian estimation based on record values and Chan (1998) presents interval estimation according to records for groups of scales and locations. Soliman and Abd Ellah (2006) compared Bayesian and Non-Bayesian estimation based on records.

The Lomax distribution plays an important role in reliability. Consider the oneparameter Lomax distribution with pdf 


$$
f(x ; \theta)=\frac{1}{\theta}(1+x)^{-\left(\frac{1}{\theta}+1\right)} \quad x \geq 0, \theta>0,
$$

and cdf

$$
F(x ; \theta)=1-(1+x)^{-\frac{1}{\theta}} \quad x \geq 0, \theta>0 .
$$

An application of the Lomax distribution in receiver operating characteristic (ROC) was presented by Campbell and Ratnaparkhi (1993). Distributional properties and recurrence relation moments of record values was studied by Balakrishnan (1994) and Ahsanullah (1991). Much work has been done with respect to estimating the parameters using both classical and Bayesian techniques, and parametric and nonparametric inference based on record values have also been studied extensively (for example, see Ahmadia, et al., 2009; Soliman \& AlAbound, 2008; Baklizi, 2008).

This study has several components: It considers Lomax parameter estimation based on record values. It estimates the parameter $\theta$ using maximum likelihood and method of moment (MME) based on record values. It uses an appropriate selection of density function for a prior distribution to derive a Bayesian estimation based on record values. For the latter, by applying an appropriate selection for the prior density, the society parameter is controlled; this means that the Mean Square Error MSE) of the Bayesian estimation is controlled by controlling the parameters of this distribution. Finally, it derives the shortest interval estimation and Highest Posterior Density (HPD) interval estimation based on record values. Examples are used to illustrate the various components.

Point Estimation of $\theta$ Parameter: The Method of Maximum Likelihood Estimation

If $X_{T(1)}, X_{T(2)}, \ldots, X_{T(n)}$ represents the first $n$ upper record values from the Lomax distribution in (1), then the joint distribution of $X_{T(1)}, X_{T(2)}, \ldots, X_{T(n)}$ is
$f\left(x_{T(1)}, x_{T(2)}, \ldots, x_{T(n)}\right)=f\left(x_{T(n)} ; \theta\right) \prod_{i=1}^{n-1} h\left(x_{T(i)} ; \theta\right)$,

where

$$
h\left(x_{T(i)} ; \theta\right)=\frac{f\left(x_{T(i)}\right)}{1-F\left(x_{T(i)}\right)} .
$$

Thus, for the Lomax distribution,

$$
f\left(x_{T(1)}, x_{T(2)}, \ldots, x_{T(n)}\right)=\theta^{-n} \frac{\left(1+x_{T(n)}\right)^{-\frac{1}{\theta}}}{\prod_{i=1}^{n}\left(1+x_{T(i)}\right)},
$$

and the log likelihood function is

$$
L=-n \ln \theta-\left(\frac{1}{\theta}\right) \ln \left(1+x_{T(n)}\right)-\sum_{i=1}^{n} \ln \left(1+x_{T(i)}\right) .
$$

The maximum likelihood estimation (MLE) based on records can be obtained from (4) as

$$
-\frac{n}{\theta}+\frac{\ln \left(1+x_{T(n)}\right)}{\theta^{2}}=0
$$

and

$$
\hat{\theta}=\frac{\ln \left(1+x_{T(n)}\right)}{n} .
$$

Using (2), the marginal pdf of $X_{T(n)}$ can be derived as

$$
\begin{aligned}
& f\left(x_{T(n)}\right)= \\
& \frac{1}{\theta^{n}(n-1) !}\left(1+x_{T(n)}\right)^{-\left(\frac{1}{\theta}+1\right)}\left(\ln \left(1+x_{T(n)}\right)\right)^{n-1},
\end{aligned}
$$

therefore,

$$
\left(\hat{\theta}_{M L E}\right)=\theta, \operatorname{Var}\left(\hat{\theta}_{m l e}\right)=\frac{\theta^{2}}{n} .
$$

conversely, if (3) is rewritten as 


$$
\begin{aligned}
& f\left(x_{T(1)}, x_{T(2)}, \ldots, x_{T(n)}\right)= \\
& \exp \left(-n \ln \theta-\frac{1}{\theta} \ln \left(1+x_{T(n)}\right)-\sum_{i=1}^{n} \ln \left(1+x_{T(i)}\right)\right),
\end{aligned}
$$

then $\ln \left(1+x_{n}\right)$ is a complete sufficient statistic for parameter $\theta$. Therefore $\hat{\theta}_{M L E}$ based on record is the equal to the Uniformly MinimumVariance Unbiased Estimator (UMVUE) for parameter $\theta$.

Point Estimation of $\theta$ Parameter: The Method of Moment Estimation

The MME, first introduced by Pearson (1894), was one of the first methods used to estimate the society parameter $\theta$ (for additional details and an example see Pearson, 1894). The Lomax parameter $\theta$ is estimated by the MME based on record values by using the density function (6), which results in

$$
E\left(X_{T(n)}\right)=\frac{1}{(1-\theta)^{n}}-1 .
$$

Next, solving the equation

$$
E\left(X_{T(n)}\right)=\bar{X}
$$

yields a MME based on record values, where $\bar{X}$ is average of the $n$ first records $\left(X_{T(1)}, X_{T(2)}, \ldots, X_{T(n)}\right)$. Thus,

$$
\hat{\theta}_{M M E}=1-\frac{1}{(1+\bar{X})^{\frac{1}{n}}} .
$$

\section{Bayesian Estimation of $\theta$ Parameter}

The Bayesian estimator of $\theta$ is obtained based on record values under the two following loss functions:

$$
L(\hat{\theta}, \theta)=\left(\frac{\hat{\theta}}{\theta}-1\right)^{2},
$$

$$
L(\hat{\theta}, \theta)=(\hat{\theta}-\theta)^{2},
$$

where $\hat{\theta}$ is an estimator of $\theta$. Assuming an inverse Weibull distribution $\operatorname{IWD}(\gamma, \beta, c=1)$, the prior for $\theta$ is conjugated as

$$
\pi(\theta)=\frac{1}{\Gamma(\gamma) \beta}\left(\frac{\beta}{\theta}\right)^{\gamma+1} \exp \left(-\frac{\beta}{\theta}\right),
$$

such that

$$
\begin{aligned}
& E(\theta)=\frac{\beta}{\gamma-1}, \\
& \operatorname{Var}(\theta)=\frac{\beta^{2}}{(\gamma-1)^{2}(\gamma-2)}
\end{aligned}
$$

where $\quad \gamma>0, \beta>0 . \quad$ Note that $\frac{1}{\theta} \sim \operatorname{gamma}(\gamma, \beta)$. This prior density has an advantage over other priors because it is easy to use and the parameter $(\gamma, \beta)$ can be chosen such that prior precision for the true value of $\theta$ is fulfilled because Bayesian estimations are functions of $(\gamma, \beta)$, therefore, the precision of the Bayesian estimations cannot be controlled by altering the prior distribution parameters. Combining likelihood function (3) with prior density (9), the posterior density of $\theta$ is obtained as

$$
\begin{aligned}
& \pi(\theta \mid x)= \\
& \left\{\begin{array}{l}
\frac{1}{\Gamma(n+\gamma)\left[\ln \left(1+x_{T(n)}\right)+\beta\right]}\left(\frac{\ln \left(1+x_{T(n)}\right)+\beta}{\theta}\right)^{n+\gamma 1} \\
\times \exp \left(-\frac{\ln \left(1+x_{T(n)}\right)+\beta}{\theta}\right)
\end{array}\right\}
\end{aligned}
$$

where $x=x_{T(1)}, \ldots, x_{T(n)}, \theta>0$. Note that

and 
$\left(\frac{1}{\theta} \mid x_{T(1)}, \ldots, x_{T(n)}\right) \sim \operatorname{gamma}\left(n+\gamma, \beta+\operatorname{Ln}\left(1+x_{T(n)}\right)\right)$.

Bayesian Estimator of $\theta$ Under Quadratic Loss Function

The posterior distribution of $\theta$ is $\operatorname{IWD}\left(n+\gamma, \beta+\ln \left(1+x_{n}\right), c=1\right)$, where IWD is Inverse Weibull Distribution (in other words $I W D=\frac{1}{W}$ where $\mathrm{W}$ is a Weibull variable) and the Bayes estimator of $\theta$ is based on record values under a quadratic loss function (7), for example $\hat{\theta}_{b, 1}$, as given by Berger (1985) is

$$
\begin{aligned}
\hat{\theta}_{b, 1}= & \frac{E\left(\omega(\theta) \mu(\theta) \mid X_{T(1)}, X_{T(2)}, \ldots, X_{T(n)}\right)}{E\left(\omega(\theta) \mid X_{T(1)}, X_{T(2)}, \ldots, X_{T(n)}\right)} \\
= & \frac{E\left(\frac{1}{\theta} \mid X_{T(1)}, X_{T(2)}, \ldots, X_{T(n)}\right)}{E\left(\frac{1}{\theta^{2}} \mid X_{T(1)}, X_{T(2)}, \ldots, X_{T(n)}\right)} \\
= & \frac{\beta+L n\left(1+x_{T(n)}\right)}{n+\gamma+1} .
\end{aligned}
$$

Bayesian Estimator of $\theta$ Under Squared Error Loss Function

Considering the posterior distribution of $\theta$ and loss function (8), the Bayes estimator based on record values, for example, $\hat{\theta}_{b, 2}$, is given as (Berger, 1985):

$$
\begin{aligned}
\hat{\theta}_{b, 2} & =E\left(\mu(\theta) \mid X_{T(1)}, X_{T(2)}, \ldots, X_{T(n)}\right) \\
& =E\left(\theta \mid X_{T(1)}, X_{T(2)}, \ldots, X_{T(n)}\right) \\
& =\frac{\operatorname{Ln}\left(1+x_{T(n)}\right)+\beta}{n+\gamma-1} .
\end{aligned}
$$

As a result, the Bayesian estimation is formed as a differentia combination of both prior distribution and sample distribution as:

$$
\hat{\theta}_{b, 2}=\frac{n}{n+\gamma-1} \frac{\ln \left(1+x_{T(n)}\right)}{n}+\frac{\gamma-1}{n+\gamma-1} \frac{\beta}{\gamma-1} .
$$

Interval Estimation of $\theta$ Based on Record Values: The Shortest Interval Estimation

To obtain the shortest $(1-\alpha) \%$ confidence interval estimation based on record values, a pivot quantity is chosen as a function of a minimal sufficient statistic for parameter $\theta\left(\hat{\theta}_{M L E}\right)$ such that

$$
Q=\frac{2 \ln \left(1+X_{T(n)}\right)}{\theta}
$$

From (6) it is clear that the distribution of $Q$ is $\chi_{2 n}$ for any constants $a$ and $b$, hence,

$$
\begin{aligned}
P(a<Q<b) & =\int_{a}^{b} f_{Q}(t) d t \\
& =1-\alpha .
\end{aligned}
$$

Algebraic manipulation results in the confidence interval

$$
\frac{2 \ln \left(1+x_{T(n)}\right)}{b}<\theta<\frac{2 \ln \left(1+x_{T(n)}\right)}{a},
$$

thus, the length of interval is obtained as

$$
L=2 \ln \left(1+x_{T(n)}\right)\left[\frac{1}{a}-\frac{1}{b}\right]
$$

To minimize (13) and satisfy (12), $a$ and $b$ are selected using the Lagrange multipliers method

$\psi(a, b, \lambda)=$

$$
2 \ln \left(1+x_{T(n)}\right)\left(\frac{1}{a}-\frac{1}{b}\right)+\lambda\left(\int_{a}^{b} f_{Q}(t) d t-(1-\alpha)\right) .
$$

After derivation by $\lambda, a$, and $b$, the following results: 


$$
\left\{\begin{array}{c}
\int_{a}^{b} f_{Q}(t) d t=1-\alpha \\
\frac{-2 \ln \left(1+x_{T(n)}\right)}{a^{2}}-\lambda f(a)=0 \Rightarrow\left\{\begin{array}{l}
\int_{a}^{b} f_{Q}(t) d t=1-\alpha \\
a^{2} f_{Q}(a)=b^{2} f_{Q}(b)
\end{array}\right. \\
\frac{2 \ln \left(1+x_{T(n)}\right)}{b^{2}}+\lambda f(b)=0
\end{array}\right.
$$

Accordingly, $a$ and $b$ must satisfy (14) to yield the shortest interval estimation for $\theta$ :

$$
\begin{aligned}
P(a<Q<b) & =\int_{a}^{b} f_{Q}(t) d t \\
& =1-\alpha, a^{2} f_{Q}(a) \\
& =b^{2} f_{Q}(b)
\end{aligned}
$$

Interval Estimation of $\theta$ Based on Record Values: Highest Posterior Density (HPD) $\theta$ Estimation

After obtaining the posterior distribution $\pi\left(\theta \mid X_{T(1)}, X_{T(2)}, \ldots, X_{T(n)}\right)$, the problem of the likelihood that the parameter $\theta$ lies within the interval $\left[c_{L}, c_{U}\right]$ arises. Bayesians call the interval based on the posterior distribution a credible interval; the interval $\left[c_{L}, c_{U}\right]$ is said to be a $(1-\alpha) \%$ credible interval for $\theta$ if

$$
\int_{c_{L}}^{c_{U}} \pi\left(\theta \mid X_{T(1)}, X_{T(2)}, \ldots, X_{T(n)}\right) d \theta=1-\alpha .
$$

The Highest Posterior Density (HPD) region is given by $\left\{A: \pi\left(\theta \mid X_{T(1)}, X_{T(2)}, \ldots, X_{T(n)}\right) \geq c\right\}$ where $\mathrm{c}$ is chosen so that

$$
\begin{gathered}
\int_{c_{L}}^{c_{U}} \pi\left(\theta \mid X_{T(1)}, X_{T(2)}, \ldots, X_{T(n)}\right) d \theta=1-\alpha \\
\pi\left(c_{L} \mid X_{T(1)}, X_{T(2)}, \ldots, X_{T(n)}\right)=\pi\left(c_{U} \mid X_{T(1)}, X_{T(2)}, \ldots, X_{T(n)}\right)
\end{gathered}
$$

The HPD interval estimation is optimal in the sense that it results in the shortest interval. Let $\lambda=\frac{1}{\theta}$, by this assumption the posterior distribution of $\lambda$ is Gamma $\left(n+\gamma, \ln \left(1+x_{n}\right)+\beta\right) . \quad$ After algebraic manipulation, an HPD estimation $(1-\alpha) \%$ for parameter $\theta$ based on records is given by

$$
\begin{aligned}
\frac{\Gamma^{*}\left(n+\gamma, A c_{L}, A c_{U}\right)}{\Gamma(n+\gamma)} & =1-\alpha,\left(\frac{c_{L}}{c_{U}}\right)^{n+\gamma-1} \\
& =\exp \left(c_{L} A-c_{U} A\right),
\end{aligned}
$$

where $A=\beta+\ln \left(1+x_{T(n)}\right)$ and $\Gamma^{*}$ is the generalized incomplete Gamma function. Therefore HPD interval estimation based on record values can be obtained as:

$$
\theta \in\left[\frac{1}{c_{U}}, \frac{1}{c_{L}}\right]
$$

MSE and Bias

Simulation and Examples

To illustrate the estimation techniques developed, consider the following simulated data from the Lomax distribution:

\begin{tabular}{|c|c|c|}
\hline 3.286379 & 2.652416 & 1.325698 \\
\hline 1.895476 & 16.420820 & 10.123657 \\
\hline 1.254875 & 14.852147 & 12.985314 \\
\hline 11.684235 & 15.365742 & 1085.950045 \\
\hline 50.254198 & 850.569874 & 32.154875 \\
\hline 950.548796 & 2423.065086 & 1.989562 \\
\hline 84.254187 & 1240.325487 & 7372.085167 \\
\hline 2.658474 & 352.325469 & 6524.123548 \\
\hline 15.987455 & 33.659874 & 5487.214587 \\
\hline 1.235478 & 3658.125489 & 9083.239327 \\
\hline 48.236584 & 6985.125489 & 6.325698 \\
\hline 448.125634 & 8754.215487 & 47739.689056 \\
\hline 125.258643 & 25.365987 & 12543.2158746 \\
\hline 25413.125487 & 256.326598 & 1254.365241 \\
\hline 1.36548 & 16845.362545 & 25.326874 \\
\hline 6985.125469 & 7.365214 & 121942.356923 \\
\hline
\end{tabular}


This data was obtained by using the transformation $x_{i}=\frac{1}{\left(1-u_{i}\right)^{\theta}}-1$, where $u_{i}$ is a uniformly distributed random variable. If only the upper record values have been observed, these are:

\begin{tabular}{|c|c|}
\hline 3.286379 & 7372.085167 \\
\hline 16.420820 & 9083.239327 \\
\hline 1085.950045 & 47739.689056 \\
\hline 2423.065086 & 121942.356923 \\
\hline
\end{tabular}

for a non-informative prior distribution with $\gamma=0, \beta=1$, and $\gamma=8, \beta=7.56$ for an informative prior distribution. Results from equations (5), (10) and (11) for the parameter $\theta$ computed for $n=4,5,6,7,8$ are presented in Table1.

Interval Estimation

Results from using equations (14) and (15) for the parameter, $\lambda=\frac{1}{\theta}$ computed for $n=4,5,6,7,8$ are presented in Tables 2 and 3 .

Table 1: Estimation, Bias and MSE

\begin{tabular}{|c|c|c|c|c|}
\hline $\begin{array}{c}\text { Number of } \\
\text { Records }(\mathrm{n}=)\end{array}$ & & Estimate & Bias & MSE \\
\hline 4 & & 1.948300 & 0 & 0.9489683 \\
5 & & 1.781118 & 0 & 0.6344765 \\
6 & $\hat{\theta}_{M L E}$ & 1.519049 & 0 & 0.3845852 \\
7 & & 1.539077 & 0 & 0.3383940 \\
8 & & 1.463914 & 0 & 0.2678805 \\
\hline 4 & & 1.758640 & -0.18966006 & 0.6433108 \\
5 & & 1.650932 & -0.13018638 & 0.4575572 \\
6 & $\hat{\theta}_{b, 1}$ & 1.444899 & -0.07414991 & 0.2880506 \\
7 & Non-Informative & 1.471692 & -0.06738463 & 0.2636236 \\
8 & & 1.412368 & -0.05154600 & 0.2143157 \\
\hline 4 & & 2.931067 & 0.9827668 & 2.6528857 \\
5 & & 2.476398 & 0.6952796 & 1.4747832 \\
6 & $\hat{\theta}_{b, 2}$ & 2.022859 & 0.5308099 & 0.8076270 \\
7 & Non-Informative & 1.962257 & 0.4231795 & 0.6396728 \\
8 & & 1.815902 & 0.3519877 & 0.4737801 \\
\hline 4 & & 1.181015 & -0.7672848 & 0.6785692 \\
5 & & 1.176114 & -0.6050046 & 0.4469587 \\
6 & $\hat{\theta}_{b, 1}$ & 1.111620 & -0.4074296 & 0.2275325 \\
7 & Informative & 1.145846 & -0.3932308 & 0.2194012 \\
8 & & 1.133607 & -0.3303074 & 0.1684260 \\
\hline 4 & & 1.395746 & -0.5525547 & 0.4308002 \\
5 & & 1.372133 & -0.4089857 & 0.2774215 \\
7 & & 1.282638 & -0.2364112 & 0.1378137 \\
\hline & & 1.309539 & -0.2295385 & 0.1372864 \\
& & 1.284754 & -0.1791599 & 0.1082954 \\
\hline
\end{tabular}


NASIRI \& HOSSEINI

Table2: Shortest $(1-\alpha) \%$ Confidence Interval Estimation Based On Record Values

\begin{tabular}{|c|c|c|c|c|}
\hline $\begin{array}{c}\text { Number of } \\
\text { Records } \\
(\mathrm{n}=)\end{array}$ & \multicolumn{2}{|c|}{ Interval } & Length & $\begin{array}{c}(1-\alpha) \% \\
\text { Confidence }\end{array}$ \\
\hline 4 & 0.7071710 & 4.549181 & 3.842010 & \\
5 & 0.7314714 & 3.743103 & 3.011632 & \\
6 & 0.6822589 & 2.965349 & 2.283090 & $90 \%$ \\
7 & 0.7401722 & 2.843073 & 2.102900 & \\
8 & 0.7432804 & 2.589680 & 1.846400 & \\
\hline 4 & 0.6255906 & 5.766975 & 5.141384 & \\
5 & 0.6532331 & 4.580130 & 3.930780 & \\
6 & 0.6139227 & 3.545798 & 2.931875 & $95 \%$ \\
7 & 0.6765692 & 3.340115 & 2.669904 & \\
8 & 0.6765692 & 3.001246 & 2.324677 & \\
\hline 4 & 0.5012662 & 9.505643 & 9.003677 & \\
5 & 0.5321775 & 7.002903 & 6.470725 & \\
6 & 0.5064018 & 5.142492 & 4.636090 & $99 \%$ \\
7 & 0.5586008 & 4.663650 & 4.104764 & \\
8 & 0.5689107 & 4.069324 & 3.500414 & \\
\hline
\end{tabular}

Table3: Highest Posterior Distribution (HPD) $(1-\alpha) \%$ Interval Estimation Based On Record Statistics

\begin{tabular}{|c|c|c|c|c|c|c|}
\hline $\begin{array}{c}\text { Number of } \\
\text { Records } \\
(\mathrm{n}=)\end{array}$ & \multicolumn{2}{|c|}{$\lambda \in\left(\lambda_{L}, \lambda_{U}\right)$} & \multicolumn{2}{c|}{$\theta \in\left[\frac{1}{\lambda_{U}}, \frac{1}{\lambda_{L}}\right]$} & Length & $\begin{array}{c}(1-\alpha) \% \\
\text { Confidence }\end{array}$ \\
\hline 4 & 0.414500 & 1.137700 & 0.878966 & 2.412545 & 1.533579 & \\
5 & 0.430000 & 1.140900 & 0.876501 & 2.325581 & 1.449080 & \\
6 & 0.472900 & 1.196200 & 0.835980 & 2.114612 & 1.278631 & $90 \%$ \\
7 & 0.467300 & 1.164000 & 0.859100 & 2.139900 & 1.280846 & \\
8 & 0.483600 & 1.173200 & 0.852369 & 2.067825 & 1.215455 & \\
\hline 4 & 0.3668000 & 1.237600 & 0.808015 & 2.726281 & 1.918266 & \\
5 & 383300 & 1.236500 & 0.808734 & 2.608923 & 1.800188 & \\
6 & 0.423500 & 1.293800 & 0.772917 & 2.361275 & 1.588358 & $95 \%$ \\
7 & 0.421100 & 1.254100 & 0.797384 & 2.374733 & 1.577384 & \\
8 & 0.437700 & 1.260800 & 0.793147 & 2.284670 & 1.491523 & \\
\hline 4 & 0.295500 & 1.416700 & 0.705865 & 3.384095 & 2.678229 & \\
5 & 0.312600 & 1.407700 & 0.710378 & 3.198976 & 2.488598 & \\
6 & 0.348500 & 1.468000 & 0.681198 & 2.869400 & 2.188242 & $99 \%$ \\
7 & 0.350100 & 1.415400 & 0.706514 & 2.856327 & 2.149813 & \\
8 & 0.366900 & 1.417600 & 0.705417 & 2.725538 & 2.020121 & \\
\hline
\end{tabular}


Figure 1: MSE's of the Estimators $\hat{\theta}_{M L E}, \hat{\theta}_{b, 1}$, and $\hat{\theta}_{b, 2}$ Informative and Non-Informative MSE's of the MLE and Bayesian estimator based on

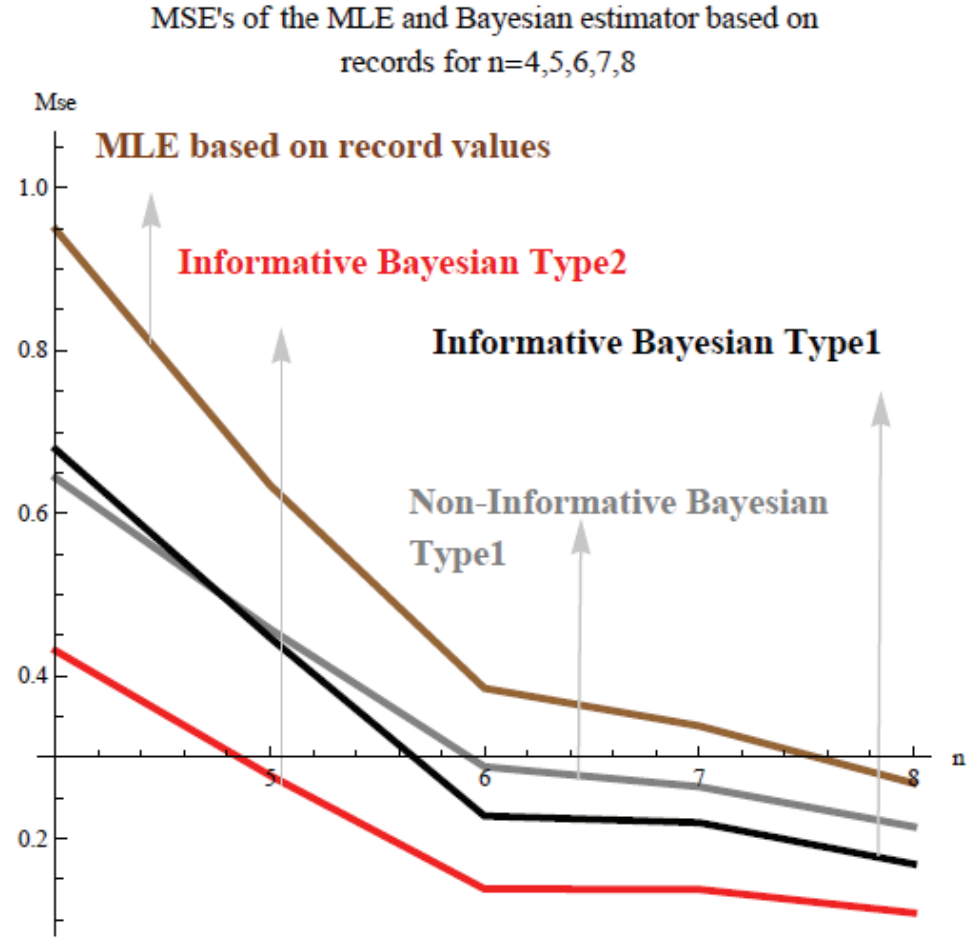

Figure 2: Lengths of the Shortest Interval and Highest Posterior Distribution (HPD) Estimations Based on Record Statistics for $90 \%$ Confidence for $n=4,5,6,7,8$ and confidence $=90$

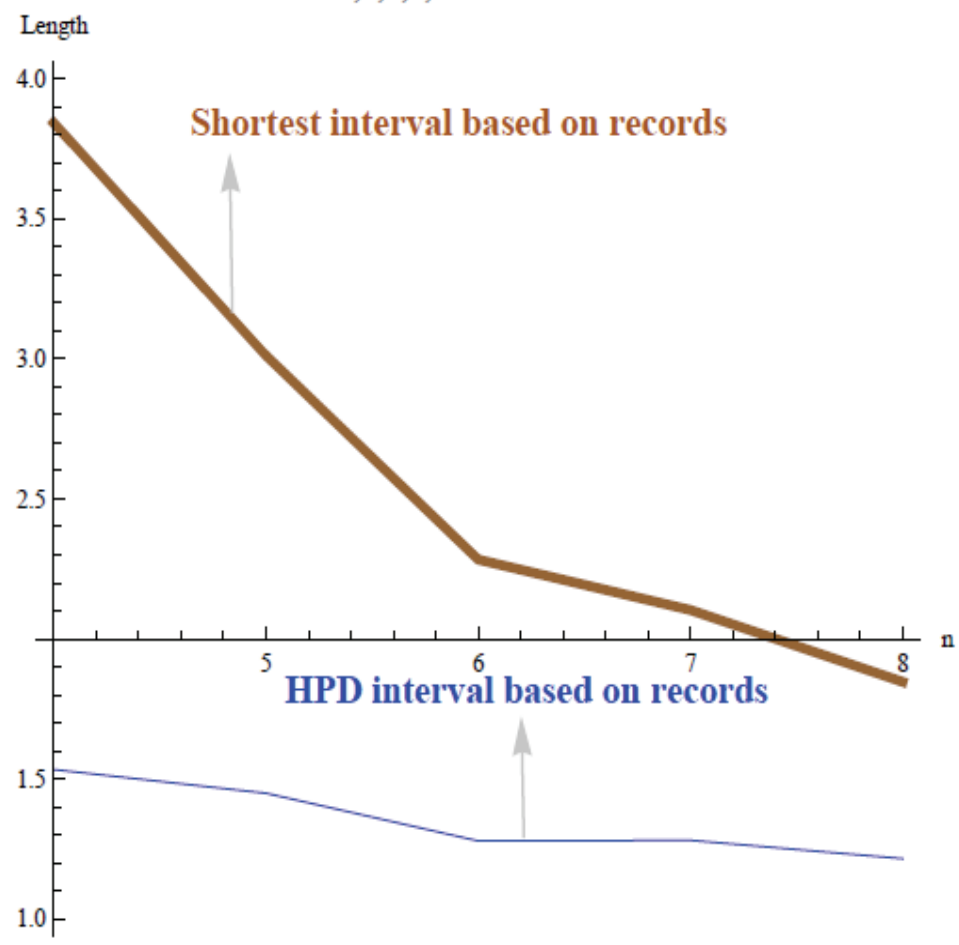


NASIRI \& HOSSEINI

Figure 3: Lengths of the Shortest Interval and Highest Posterior Distribution (HPD) Estimations Based on Record Statistics for 95\% Confidence

Lengths of the Shortest and HPD interval based on records

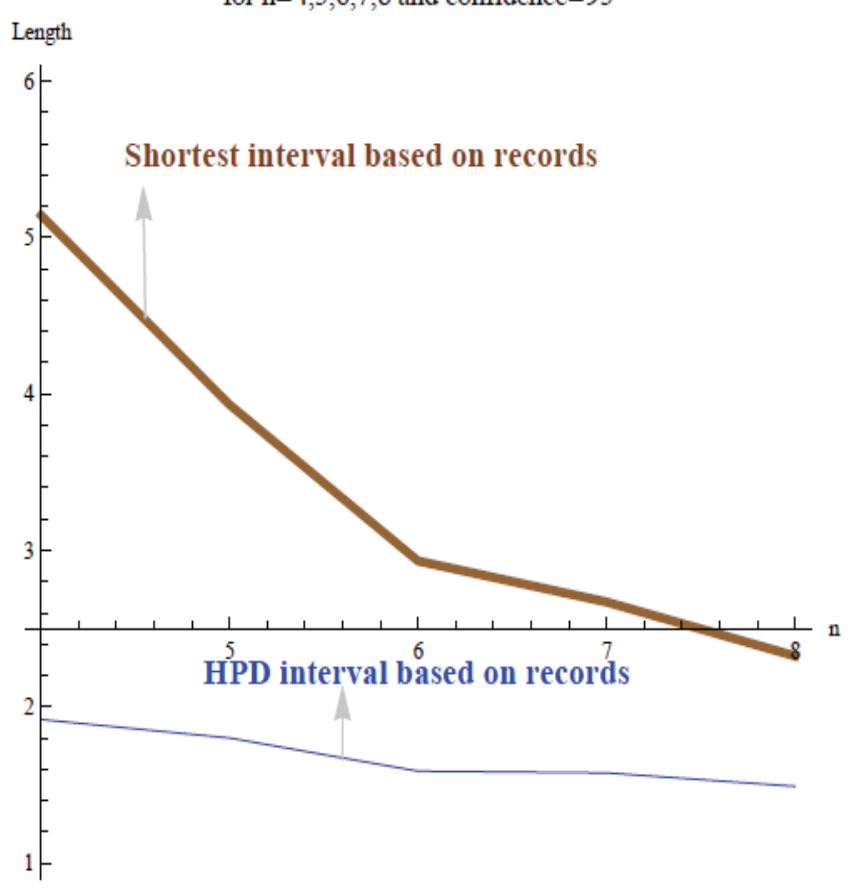

Figure 4: Lengths of the Shortest Interval and Highest Posterior Distribution (HPD) Estimations Based on Record Statistics for $99 \%$ Confidence

Lengths of the Shortest and HPD interval based on records

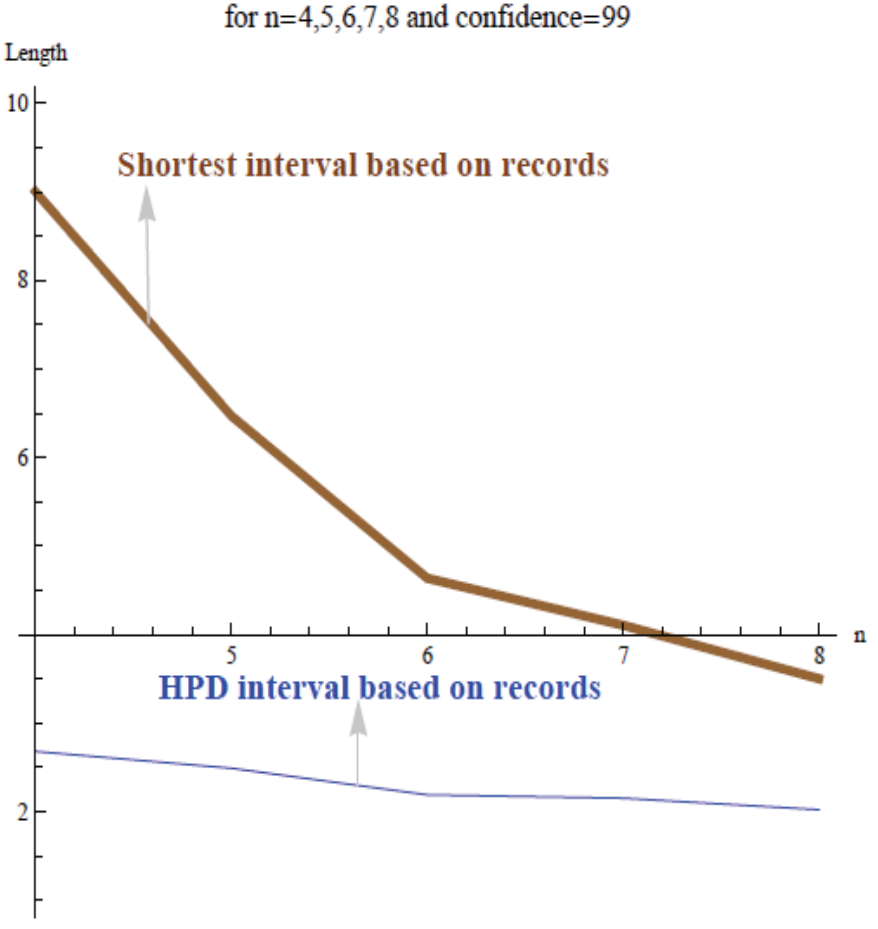


Figure 5: Lengths of the Shortest Interval and Highest Posterior Distribution (HPD) Estimations Based on

Record Statistics for $90 \%, 95 \%$ and $99 \%$ Confidence

Lengths of the Shortest and HPD interval based on records

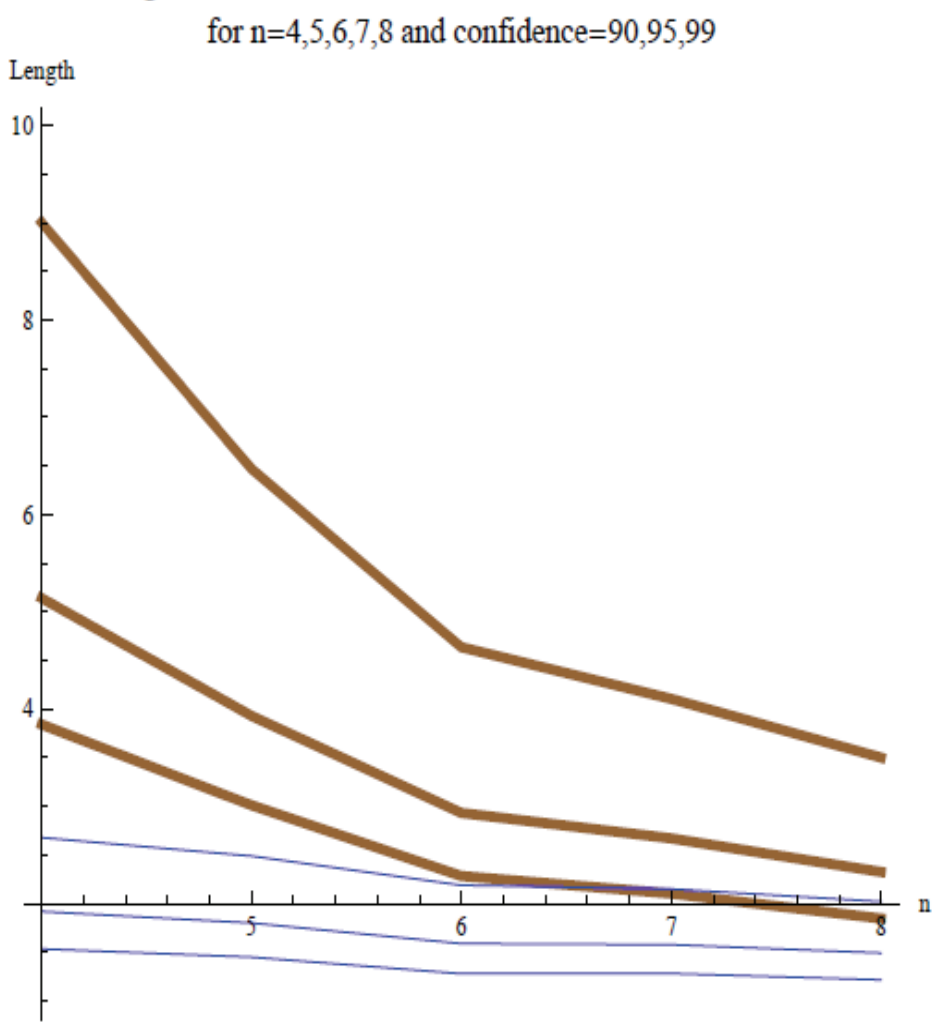

\section{Conclusion}

MLE and Bayesian estimations based on record values were obtained. For the Bayes estimations, in order to control the passive parameter of society, the prior distribution was assumed to be Gamma. In addition, Bayes estimations were obtained for two types of loss functions and, with a view of prior estimation, using an informative posterior density function, HPD estimations were obtained in a theoretic way (see Table 3). Conversely, the shortest confidence interval was obtained using a MLE based on records and equation (14) (Tate \& Klett, 1959); see Table 2 for results.

Theoretical results of the study are explained numerically by simulation in the following ways: Table 1 shows that an informative Bayesian estimation based on records under squared error loss function has the lowest MSE compared to the informative Bayesian estimation, which is based on records under a quadratic loss function with a noninformative Bayesian estimation under a squared error loss function.. This is also compared to a MLE based on records; comparisons are shown in Figure 1. Confidence intervals and their lengths for record numbers $4,5,6,7,8$ and confidence levels $90 \%, 95 \%$ and $99 \%$ were obtained. The longer the $n$, the shorter the interval distance (see Table 3). Comparing Tables 2 and 3, it the point at which HPD estimations have a shorter length than the confidence interval with optimal length is observed. This comparison is illustrated in Figures 2, 3 and 4 for various confidence levels; Figure 5 shows the comparison for all levels. 
Acknowledgments

The authors are thankful to the referees and editor for their valuable comments. This work was partially supported by the Tehran Payame Noor University (PNU) through a grant.

\section{References}

Ahmadia, J., Jozanib, M. J., Marchandc, E., \& Parsian, A. (2009). Bayes estimation based on $k$-record data from a general class of distributions under balanced type loss functions. Journal of Statistical Planning and Inference, 139, 1180-1189.

Ahmed A., Soliman, A. H., Abd Ellah, K. S. Sultan. (2006). Comparison of estimates using record statistics from Weibull model: Bayesian and non-Bayesian approaches. Computational Statistics \& Data Analysis, 51, 2065-2077.

Ahsanullah, M. (1991). Record values of the Lomax distribution. Statistica Neerlandica, 45, 21-29.

Ahsanullah, M. (1998). Introduction to record values. Needham Heights, MA: Ginn Press.

Ahsanullah, M., \& Holland, B. (1994). On the use of record values to estimate the location and scale parameters of the generalized extreme value distribution. The Indian Journal of Statistics, 56, 480-499.

Arnold, B. C., Balakrishnan, N., \& Nagaraja, H. N. (1998). Records. Canada: Wiley.

Asgharzadeh, A. (2009). On Bayesian estimation from exponential distribution based on records. Journal of the Korean Statistical Society, 38, 125-130.

Baklizi, A. (2008). Likelihood and Bayesian estimation of $\operatorname{Pr}(\mathrm{X}<\mathrm{Y})$ using lower record values from the generalized exponential distribution. Computational Statistics and Data Analysis, 52, 3468-3473.
Balakrishnan, M. (1994). Relations for single and product moments of record values from Lomax distribution. The Indian Journal of Statistics, 56, 140-146.

Berger, J. O. (1985). Statistical decision theory and Bayesian analysis. New York, Springer.

Campbell, G., \& Ratnaparkhi, M. V. (1993). An application of Lomax distribution in receiver operating characteristic (ROC) curve analysis. Communications in Statistics, 22, 1681-1687.

Chan, P. S. (1998). Interval estimation of location and scale parameters based on record values, Statistics \& Probability Letters, 37, 4958.

Mohammad, Z. R. (2002). Inferences for generalized exponential distribution Based on record statistics. Journal of Statistical Planning and Inference, 104, 339-350.

Nevzorov, V. B. (2001). Records: Mathematical theory. USA: American Mathematical Society.

Pearson, K. (1894). Contributions to the mathematical theory of evolution. Phil. Trans. Royal Soc.

Sevgi, Y. O., Mohammad, A., Fazil A. A., \& Funda, A. (2005).Switching record and order statistics via random contractions. Statistics \& Probability Letters, 73, 207-217.

Soliman, A. A., \& Al-Abound, F. M. (2008). Bayesian inference using record values from Rayleigh model with application. European Journal of Operational Research, 185, 659-672.

Tate, \& Klett. (1959). Optimal confidence intervals for the variance of a normal distribution. Journal of the American Statistical Association, 54(287), 674-682. 